Circulating sclerostin associated with vertebral bone marrow fat in older men but not women

V. Ma ${ }^{1}$, X. Li ${ }^{1}$, S. Sigurdsson ${ }^{2}$, G. Eriksdottir ${ }^{2}$, A.M. Hauksdottir ${ }^{2}$, L. Palermo ${ }^{1}$, T. Hue ${ }^{1}$, T. Lang ${ }^{1}$, T.B. Harris ${ }^{3}$, C.J. Rosen ${ }^{4}$, E. Vittinghoff ${ }^{1}$, N. Napoli ${ }^{5}$, K. Siggeirsdottir ${ }^{2}$, G. Sigurdsson ${ }^{6,7}$, D. Osakrsdottiir ${ }^{2}$, V. Gudnason ${ }^{2,6}$, A. Schwartz ${ }^{1}$

${ }^{1}$ University of California, San Francisco, California, USA ${ }^{2}$ Icelandic Heart Association, Kopavogur, Iceland

${ }^{3}$ Laboratory of Epidemiology, Demography, and Biometry, National Institute on Aging, Bethesda, MD, USA

${ }^{4}$ Maine Medical Center Research Institute, Scarborough, ME, USA

${ }^{5}$ Department of Endocrinology and Diabetes, University Campus Bio-Medico, Rome, Italy

${ }^{6}$ Faculty of Medicine, University of Iceland, Reykjavik, Iceland ${ }^{7}$ Landspital University Hospital, Reykjavik, Iceland

Introduction

Marrow fat (MF) and bone density (BMD) are negatively correlated. Proposed mechanisms include 1) a shift in
stem cell lineage allocation from osteoblasts towards adipocytes, and 2) an increase in osteoclast-promotin stem cell lineage allocation

- Sclerostin is expressed by osteocytes and reduces bone formation by inhibiting the Wnt/B-catenin pathway.

- Circulating sclerostin is also associated with higher BMD. However, the relationship between circulating sclerostin and MF is not known.

-

\section{PRIMARY OBJECTIVE}

To characterize the relationships between circulating sclerostin and MF, QCT measurements of hip and spine, and body composition parameters, we used data from the Iceland AGESReykjavik cohort of older adults.

Methods
COHORT
- 303 participants at AGES-Reykjavik follow-up visit had measurements of vertebral MF and hip/spine QCT.
- 137 women and 118 men were included in analyses.

\section{QUANTITATIVE COMPUTED TOMOGEAPHY (OCT)}

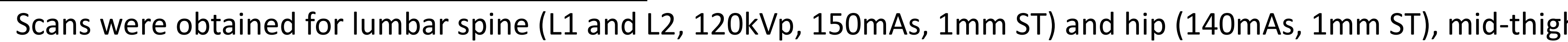
- (129kV, 1Omm ST) using 4-detector CT system (Sensation, Siemens Medical Systems, Erlangen, Germany).

- Cortical and trabecular volumetric BMDs (VBMDs) for vertebra and hip, vertebral compressive strength, hip geometry, subcutaneous fat at mid-femur and visceral fat at $L 4 / L 5$ intervertebral space, and muscle attenuation at mid-thigh were calculated from QCT dat.

PROTON MAGNETIC RESONANCE SPECTROSCOPY ( ${ }^{1} \mathrm{H}$-MRS) FOR VERTEBRAL MF

Single voxel Point Resolved Spectroscopy (PRESS) ${ }^{1} \mathrm{H}$-MRS was acquired in vertebral bodies $\mathrm{L} 1$ to $\mathrm{L} 4$ using a 1.5 Tesla scanner (GE Healthcare, Milwaukee, WI) with an eight-channel spine coil (TR/TE $=2000 / 37 \mathrm{~ms}, 64$ averages without water suppression, ,

Were calculated using GE SAGE software. The average MFs (Fat/(Fat+Water)*100\%) from all four vertebral levels were used in analyses.

DUAL-ENERGY X-RAY ABSORPTIOMETRY (DXA)

- Scans of lumbar spine, proximal femur and whole body were obtained with GE Healthcare Lunar iDXA scanner (software version 11.4)

ASSAY FOR SCLEROSTIN AND BONE TURNOVER MARKERS

- Serum was collected fasting and stored at $-70^{\circ} \mathrm{C}$.
- Sclerostin and markers for bone formation (amino-terminal propeptide of type 1 procollagen; P1NP) and bone resorption (serum C-terminal cross-linking telopeptide of type I collagen; CTX) were assayed in one batch.

\section{STATISTICAL ANALYSIS}

Least square means of selected bone and body composition parameters were compared across tertiles of serum P-values for linear trend across tertiles was used to assess association from moder.

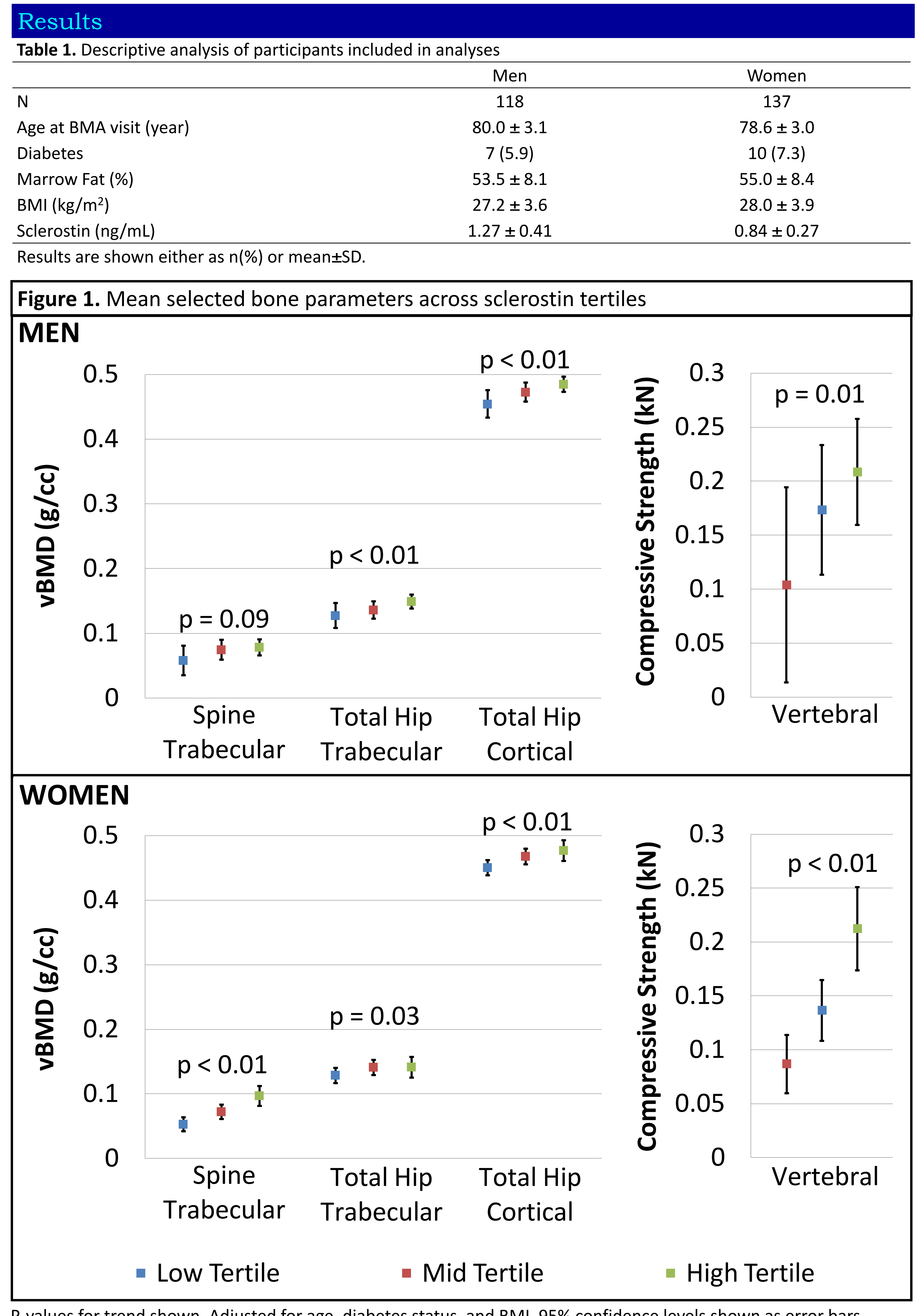

Total hip cortical and trabecular VBMD increased significantly with higher sclerostin for me and women (Figure 1).

- Spine trabecular VBMD increased significantly with higher sclerostin for women. The same positive trend, although not significant, was found in men ( $p=0.09$ ) (Figure 1 ).

- Areal BMDs measured by DXA increased significantly across sclerostin tertiles at spine femoral neck, and total hip for both men and women.

- Vetebral cross-sectional area (CSA) non-significantly increased with higher sclerostin for both men $(p=0.23)$ and women $(p=0.16)$

- PINP and CTX were negatively associated with sclerostin in women, but not men.

- MF increased significantly with higher sclerostin in men, but not women (Figure 2)

- There was a trend towards positive association between sclerostin and body mass index (BMI) in women ( $p=0.09)$, and weight in men ( $p=0.06$ )

- Total fat and lean mass measured by DXA and abdominal visceral and subcutaneous fat mass measured by QCT were not significantly associated with sclerostin levels (Figure 2).

- Thigh muscle attenuation by QCT was not significantly associated with sclerostin levels (Figure 2). However, there was a positive trend for women $(p=0.08$.

\section{Figure 2. Mean selected body composition parameters across sclerostin tertiles} MEN

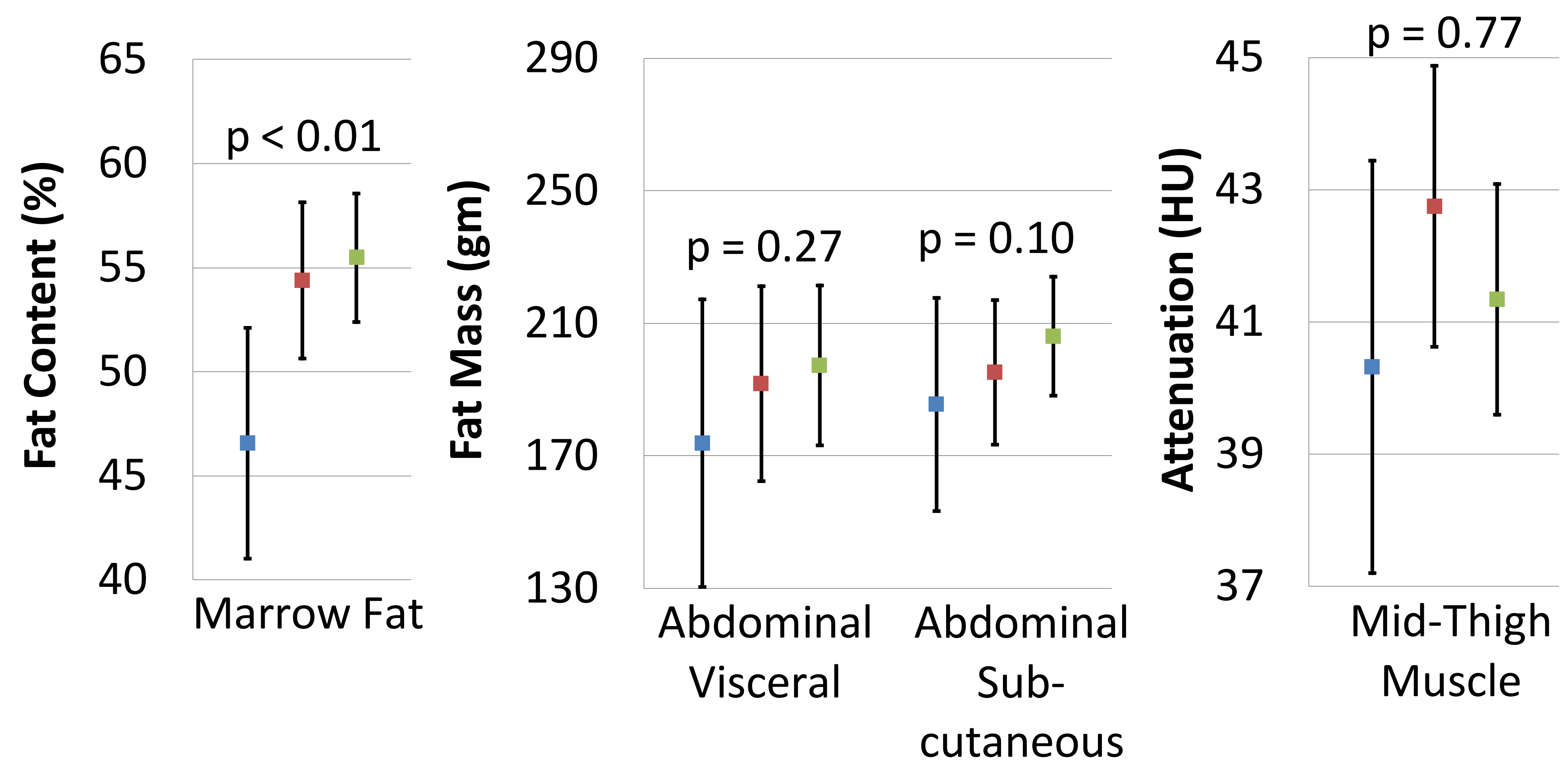

\section{WOMEN}

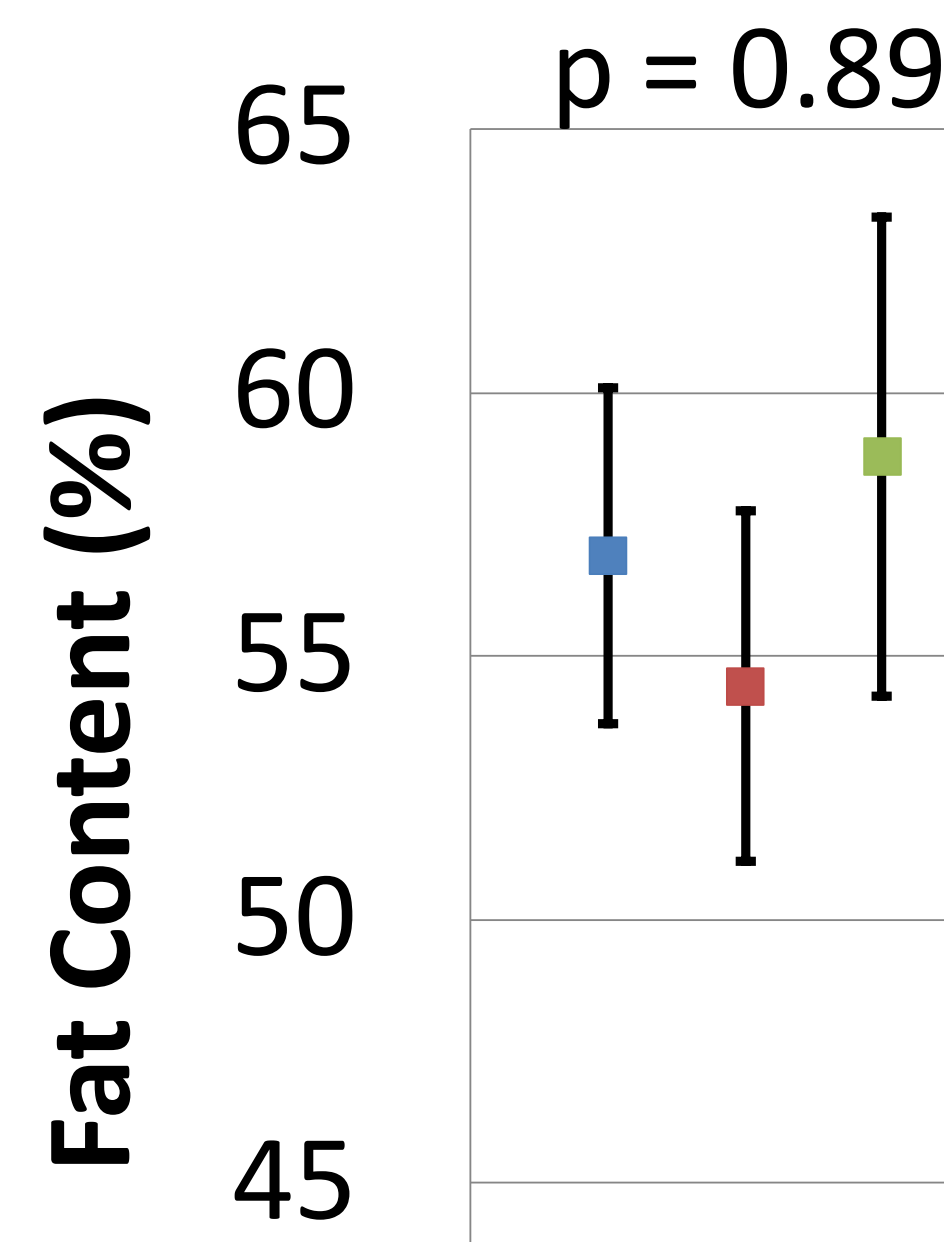

40 Marrow Fat
290

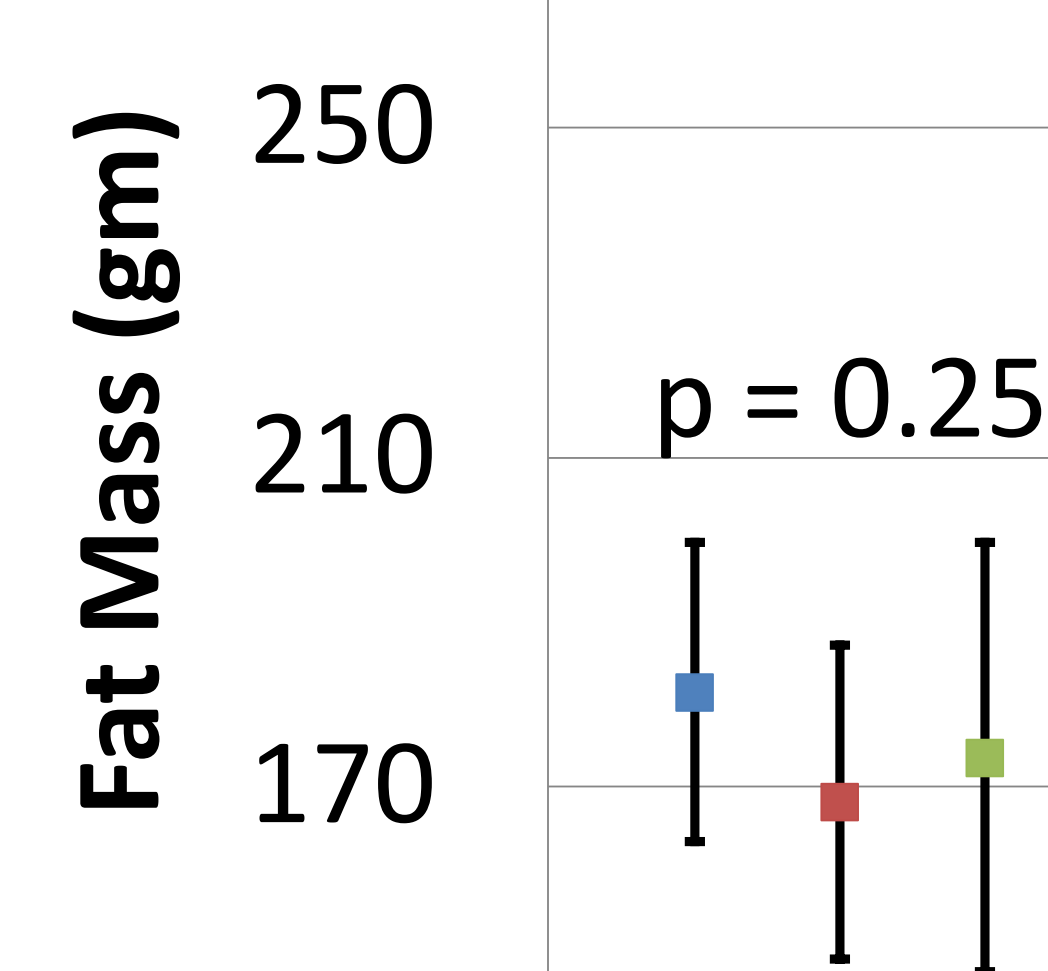

130

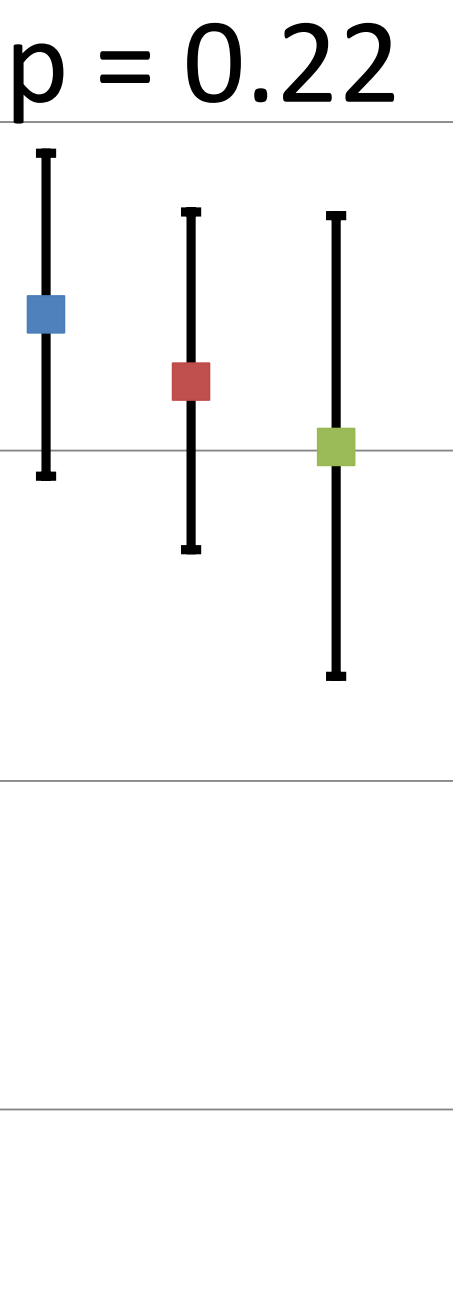

Abdominal Abdominal Sub-

cutaneous
45

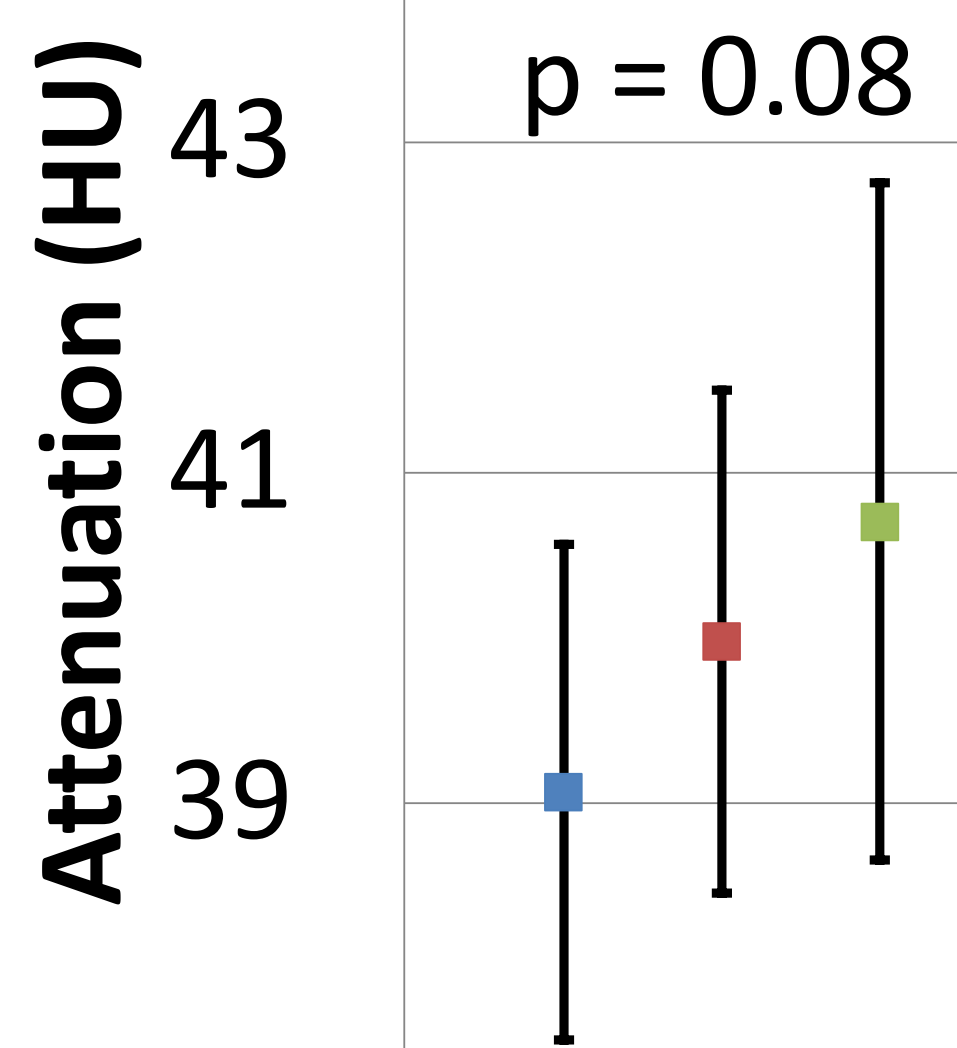

37

Mid-Thigh Muscle
- Low Tertile

- Mid Tertile

- High Tertile

- As previous report showed a negative association between MF and $\mathrm{BMD}^{1}$, it is surprising that sclerostin is positively associated with both MF and BMD in men. However, higher M was also associated with prevalent vertebral fracture in the same cohort ${ }^{1}$, independent of BMD. Sclerostin may be a marker of bone fragility, but this remains controversial.

- Previous reports on sclerostin and MF are not available. Further investigation is needed to understand the gender difference.

- Both trabecular and cortical VBMDs were positively associated with sclerostin, consisten with the only previous report on central QCT and sclerostin ${ }^{2}$

- Previous studies have reported positive ${ }^{3,4,5}$ or $\mathrm{no}^{6,7}$ association between sclerostin and weight or BMI. Another previous study found no association between sclerostin and lean mass or fat mass ${ }^{7}$. Interestingly, sclerostin increases in response to weight loss ${ }^{8}$. Longitudinal studies are needed to fully understand the relationship between sclerostin and body composition.

- A limitation of this study is that the cohort lacks racial and age diversity.

MF is positively associated with circulating sclerostin levels in men, providing additional evidence that MF and bone formation are linked.

- Circulating sclerostin is positively correlated with both trabecular and cortical vBMDs.

Schwatz AV et al. The Journal of Clinical Endocinology \& Metabolism 2013, doi: 10.1020/jc 2012-3949.

2. Modder Ul et al., Journal of Bone and Mineral Research 2011, doi: 10.1002/jbmr.217.

3. Szulc P et al., Journal of Bone and Mineral Research 2013, doi: 10.1002/jbmr.1888.

4. Sheng Z et al., Clinical Endocrinology 2012, doi: 10.1111/j.1365-2265.2011.04315.x.

5. Thorson S et al., Journal of Bone and Mineral Research 2013, doi: 10.1002/jbmr.1929.

6. Arasu A et al., The Journal of Clinical Endocrinology \& Metabolism 2012, doi: 10.1210/j. 2011-3419.

7. Amrein K et al. The Journal of Clinical Endocrinology \& Metabolism 2011, doi: 101210/jc 2011-215.

8. Armamento-Villareal R et al., Journal of Bone and Mineral Research 2012, doi: 10.1002/jbmr.1560. 